https://doi.org/10.18359/ravi.2983

\title{
Algunas tareas para evaluar la comprensión sobre el objeto matemático antiderivada $^{1}$
}

\author{
Wilson Gordillo ${ }^{2}$, Luis R. Pino-Fan ${ }^{3}$, Vicenç Font ${ }^{4}$ y Juan Carlos Ponce Campuzano 5
}

Sometido: 13 agosto de 2017

Evaluado: 02septiembre de 2017

Aceptado: 16 septiembre de 2017

\begin{abstract}
Referencia: Gordillo, W., Pino-Fan, L., Font, V. y Ponce Campuzano, J.C. (2018). Algunas tareas para evaluar la comprensión sobre el objeto matemático antiderivada. Revista Academia y Virtualidad, 11(2),1-17.
\end{abstract}

\section{Resumen}

En el presente artículo se exponen varias tareas, diseñadas para evaluar la comprensión que tienen los estudiantes de cursos de cálculo sobre el objeto matemático antiderivada. Las tareas se han creado a partir de las sugerencias y recomendaciones existentes en el amplio bagaje de investigaciones sobre didáctica del cálculo, con las que se cuenta a nivel internacional. Así mismo, se detalla el análisis ontosemiótico de cada una de las tareas propuestas, lo cual evidencia la red de objetos y procesos matemáticos -y sus vínculos-, puestos en juego en las actividades de comprensión de la antiderivada. El resultado de este estudio muestra que las tareas aquí descritas pueden ser aplicadas a estudiantes universitarios (o estudiantes de cursos de cálculo que hayan estudiado la antiderivada), y podrían ser adaptadas para abordar el estudio de dicho objeto matemático.

Palabras clave: antiderivada; comprensión; cálculo; diseño de tareas; análisis ontosemiótico

\footnotetext{
${ }^{1}$ Artículo de investigación científica y tecnológica

2 Doctor en Educación Matemática de la Universidad de Los Lagos, en Chile. Profesor Asociado de la Universidad Distrital Francisco José de Caldas. Bogotá, Colombia. Dirección postal: Carrera 7 No. 40-53, Bogotá, Colombia. Correo electrónico: wgordillot@udistrital.edu.co

${ }^{3}$ Doctor en Didáctica de la Matemática de la Universidad de Granada, en España. Profesor Asociado de la Universidad de Los Lagos, Osorno, Chile. Dirección postal: Av. Fuchslocher 1305, Osorno, Chile.

Correo electrónico: luis.pino@ulagos.cl

${ }^{4}$ Doctor en Filosofía y Ciencias de la Educación de la Universidad de Barcelona, en España. Profesor Titular de la Universidad de Barcelona. Dirección postal: Calle Figols 15, Barcelona, España.

Correo electrónico: vfont@ub.edu

${ }^{5}$ Doctor en Ciencias en la Especialidad de Matemática Educativa del Centro de Investigación y de Estudios Avanzados del Instituto Politécnico Nacional, en México. Profesor Investigador de la Universidad de Queensland, en Australia. Dirección postal: St Lucia 4072, Queensland, Australia. Correo electrónico: j.ponce@uq.edu.au
} 


\title{
Tasks for Assessing the Comprehension of the Antiderivative Mathematical Object ${ }^{1}$
}

Wilson Gordillo², Luis R. Pino-Fan ${ }^{3}$, Vicenç Font ${ }^{4}$ and Juan Carlos Ponce-Campuzano ${ }^{5}$

\section{Reference:}

Gordillo, W., Pino-Fan, L., Vicenç, F. and Ponce Campuzano, J.C (2018). "Tasks for Assessing Comprehension of the Antiderivative Mathematical Object http://cgpublisher. com/conferences/327/proposals/218/manage_workspace" Academia y Virtualidad Journal $11,(2), p-p$

\begin{abstract}
This study describes several tasks that aim to assess the comprehension of the antiderivative mathematical object by students in calculus courses. These tasks have been designed based on the suggestions and recommendations obtained from extensive studies conducted on a global scale with respect to the teaching of calculus. In addition, this study includes an ontosemiotic analysis for each of the proposed tasks, which proves the web of mathematical objects and processes as well as their interconnections that can be observed while performing different activities for assessing the student comprehension of antiderivatives. The study results denote that these tasks may be applicable to college-level courses (or to calculus courses whose syllabus includes antiderivatives) and adapted for conducting the academic study of the said mathematical object.
\end{abstract}

\section{Keywords:}

Antiderivative, Comprehension, Calculus, Task Design, Onto-Semiotic Analysis

\footnotetext{
1 A Scientific and Technological Research Paper

2 Ph.D, in Mathematics Education, University of Los Lagos, Chile. Associate Professor, Francisco José de Caldas District University. Bogotá, Colombia. Mailing Address: Carrera 7 No. 40-53, Bogotá, Colombia. E-mail: wgordillot@udistrital.edu.co

${ }^{3}$ Ph.D. in Didactics of Mathematics, University of Granada, Spain. Associate Professor, University of Los Lagos, Osorno, Chile. Mailing Address: Av. Fuchslocher 1305, Osorno, Chile. E-mail: luis.pino@ulagos.cl

${ }^{4}$ Ph.D. in Educational Sciences, University of Barcelona, Spain. Professor, University of Barcelona, Barcelona, Spain. Mailing Address: Calle Figols 15, Barcelona, Spain. E-mail: vfont@ub.edu

${ }^{5}$ Ph.D. in Science with emphasis on Educational Mathematics, Center for Research and Advanced Studies, National Polytechnic Institute. Mexico. Research Professor, University of Queensland, Queensland, Australia. Mailing Address: St Lucia 4072, Queensland, Australia. E-mail: j.ponce@uq.edu.au
} 


\section{Algumas tarefas para Avaliar a Compreensão sobre o objeto Matemático Antiderivada ${ }^{1}$}

Wilson Gordillo², Luis R. Pino-Fan ${ }^{3}$, Vicenç Font ${ }^{4}$ e Juan Carlos Ponce-Campuzano ${ }^{5}$

\section{Referência:}

Gordillo, W., Pino-Fan, L., Vicenç, F. and Ponce Campuzano, J.C (2018). “Alguns desafios para avaliar a compreensão do assunto antiderivada no cálculo matemático" Academia y Virtualidad Journal 11, (2), p-p

\section{Resumo}

No presente artigo se apresentam várias tarefas que foram projetadas para avaliar a compreensão que possuem os estudantes de cálculo sobre o objeto matemático antiderivada. As tarefas foram projetadas levando em conta as sugestões e recomendações do extenso histórico de pesquisas sobre didática de cálculo disponíveis internacionalmente. Da mesma forma, a análise ontosemiótica de cada uma das tarefas propostas é detalhada, o que evidencia a rede de objetos e processos matemáticos - e seus elos - colocados em jogo nas atividades de compreensão da antiderivada. O resultado deste estudo mostra que as tarefas descritas aqui podem ser aplicadas a estudantes universitários (ou estudantes de cursos de cálculo que tenham estudado antiderivada) e poderiam ser adaptadas para abordar o estudo do referido objeto matemático.

\section{Palavras chave:}

Antiderivada, Compreensão, Cálculo, Projetos de tarefas, Análise Ontosemiótica

\footnotetext{
${ }^{1}$ Artigo de pesquisa científica e tecnológica

${ }^{2}$ Doutor em Educação Matemática pela Universidade de Los Lagos, Chile. Professor Associado da Universidade Distrital Franisco José de Caldas, Bogotá, Colômbia. Endereço postal: Carrera 7, No. 40-53, Bogotá, Colômbia. E-mail: wgordillot@udistrital.edu.co.

${ }^{3}$ Doutor em Didática da Matemática pela Universidade de Granada, Espanha. Professor Associado da Universidade de Los Lagos, Osorno, Chile. Endereço postal: Av. Fuchslocher 1305, Osorno, Chile. E-mail: luis. pino@ulagos.cl

${ }^{4}$ Doutor em Filosofía e Ciências da Educação pela Universidade de Barcelona, Espanha. Professor Titular da Universidade de Barcelona, Barcelona, Espanha. Endereço postal: Calle Figols 15, Barcelona, Espanha. E-mail: vfont@ub.edu

${ }^{5}$ Doutor em Ciências na Especialidade de Matemática Educativa pelo Centro de Investigação e Estudos Avançados do Instituto Politécnico Nacional, México. Professor em Pesquisas na Universidade de Queensland, Queensland, Austrália. Endereço postal: St Lucia 4072, Queensland, Australia. E-mail: j.ponce@uq.edu.au
} 


\section{Introducción}

El objeto matemático antiderivada, tal como lo conocemos hoy en día, está relacionado con otras nociones del cálculo infinitesimal, tales como la derivada y la integral. Dichas nociones han sido objeto de diversas investigaciones, en las que se han producido resultados relevantes para los procesos de aprendizaje (Sfard, 1991; Tall, 1991; Artigue, 1995), para las estrategias y los procesos de enseñanza (Czarnocha, Loch, Prabhu y Vidakovich, 2001; Labraña, 2000; Robles, Del castillo y Font, 2012), y para el uso de la tecnología (Depool, 2004, Robles, Del Castillo y Font, 2012), entre otros. En el caso particular de la antiderivada, se han realizado estudios sobre la reflexión de los estudiantes al abordar reglas de integración (Metaxas, 2007), estudios históricos-epistemológicos (Gordillo y Pino-fan, 2016), y sobre el uso de la tecnología (Ponce-Campuzano y Rivera-Figueroa, 2011).

La comprensión de los objetos matemáticos en el cálculo infinitesimal ha sido abordada desde diferentes marcos teóricos de la educación matemática, como el propuesto por Sfard (1991), el cual sugiere que, a través de los ciclos mentales de interiorización, condensación y reificación, se logra la comprensión de un objeto matemático. Gray y Tall (1994), por su parte, proponen tres ciclos para la comprensión: procedimiento, proceso y preconcepto, con los cuales se logra el entendimiento de los objetos matemáticos. Kintsch (1998) utiliza un marco teórico para la comprensión de objetos matemáticos, que coincide con elementos y supuestos de la teoría de registros semióticos $\mathrm{y}$, en particular, con la contracción semiótica (Duval, 2006b). Este propone, además, abordar tareas como si fueran una fórmula, reconociendo símbolos y decodificando con el fin de dar una percepción global y situarla en un modelo adecuado para resolver y lograr comprensión. En este mismo sentido, Perie y Kieran (1994) proponen un proceso no lineal, creciente, estratificado, compuesto por ocho niveles: primitive knowing (PK), image making (IM), image having (IH), property noticing $(\mathrm{PN})$, formalising $(\mathrm{F})$, observing $(\mathrm{O})$, structuring $(\mathrm{S})$ e inventising $(\mathrm{I})$, y señalan que, una vez alcanzado el último nivel, un estudiante se puede desvincular de la situación concreta y estará preparado para efectuar lo que ellos denominan folding back; en este punto, se creará un nuevo conocimiento o se modificará uno existente.

En este artículo se presentan algunas tareas que han sido diseñadas para evaluar la comprensión de la antiderivada como objeto matemático. La propuesta que se expone en este documento, cuyo fin es evaluar la comprensión de dicho objeto matemático, toma en cuenta dos aspectos fundamentales que hoy en día parecen olvidados en la enseñanza tradicional: el acercamiento intuitivo y la conjetura - aspectos que hemos fundamentado con evidencia, obtenida a partir de un estudio histórico-epistemológico sobre la antiderivada (Gordillo y Pino-Fan, 2016) Dichos aspectos, tal como lo sustenta Tall (2009), favorecen la participación activa de un estudiante. De igual forma, de acuerdo con las posturas de Doorman y Maannen (2008), estas permiten, a su vez, dar indicaciones sobre cómo evoluciona una noción y su desarrollo conceptual.

\section{Marco referencial y metodológico}

\section{Comprensión de los objetos matemá- ticos}

Aunque, como hemos visto anteriormente, existen diversas posturas para entender la comprensión, de acuerdo con Font (2001) y con Godino, Batanero y Font (2007), hay dos maneras básicas de concebirla: como proceso mental o como competencia. Según estos autores, los dos puntos de vista responden a concepciones epistemológicas que, como mínimo, son divergentes, por no decir que están claramente enfrentadas. Los enfoques cognitivos en la didáctica de las matemáticas, en el fondo, entienden la comprensión como un proceso mental. Los posicionamientos prag- 
matistas del Enfoque Onto-Semiótico (EOS), en cambio, llevan a entender la comprensión básicamente como una competencia. Es decir, se considera que un sujeto comprende un determinado objeto matemático cuando lo usa de manera competente en diferentes prácticas.

Esta manera pragmática de entender la comprensión hace que esta se conciba también como conocimiento y aplicación de las normas que regulan una práctica. Se trata, pues, de un punto de vista que procura dilucidar la inteligibilidad de las acciones humanas, a través de la clarificación del pensamiento que las informa y situando a este en el contexto de las normas sociales y de las formas de vida dentro de las cuales aquellas ocurren. Es necesario aclarar que, dentro del EOs, enfoque teórico al que nos apegamos en este estudio, el término conocimiento se utiliza en el sentido de "constructo epistémico-cognitivo general que incluye comprensión, competencia y disposición" (Pino-Fan, Godino y Font, 2010, p. 209). La disposición o capacidad se relaciona con la noción de objeto matemático y didáctico personal, es decir, con aquello que posibilita la práctica. La competencia se relaciona con las prácticas matemáticas de los sujetos y con la activación, en dichas prácticas, de la configuración ontosemiótica cognitiva adecuada, que debería estar idóneamente acoplada a la configuración ontosemiótica epistémica de referencia (Pino-Fan, Godino y Font, 2011) y al contexto en el que se desarrolla la práctica. La comprensión, como lo afirma Pino-Fan (2014), tiene que ver con las relaciones - vistas desde la perspectiva de la congruencia matemática- que se deben establecer entre todos los elementos que intervienen en la configuración ontosemiótica cognitiva (o epistémica, en el caso de prácticas institucionales), y que activa el sujeto para resolver determinadas situaciones-problemas. En el apartado 2.3 describiremos lo que se entiende por configuración ontosemiótica (epistémica y cognitiva).

\section{Criterios para la selección de las tareas}

El diseño de cada una de las tareas busca que el objeto matemático se use de manera competente en diferentes situaciones. En el proceso de construcción de las tareas consideramos dos criterios para la selección de estas. El primer criterio estipula que los problemas o situaciones deben proporcionar información sobre el grado de ajuste del significado personal de los estudiantes respecto del significado global u holístico del objeto antiderivada (Gordillo y Pino-Fan, 2016). Para lograrlo, se incluyeron tareas que activan los diversos significados parciales de la antiderivada. El segundo criterio es que los ítems seleccionados, siguiendo la postura de investigaciones como las de Font (1999), Pino-Fan (2014) y Crisóstomo (2012), respondan a los diferentes tipos de representaciones para la antiderivada. Así mismo, como parte de este criterio, hemos considerado los resultados obtenidos de un estudio histórico-epistemológico con el cual realizamos la reconstrucción del significado holístico de la antiderivada, a partir de la caracterización de las prácticas matemáticas, representaciones, conceptos, proposiciones, procedimientos y argumentos, que se emplearon a lo largo de la historia y que dieron lugar a la emergencia, evolución, formalización y generalización de dicho objeto matemático (Gordillo y Pino-Fan, 2016). En este sentido, las tareas ponen en juego los siguientes tipos de representaciones para la antiderivada - y también para las funciones asociadas a esta-: descripción verbal, gráfica, fórmula (simbólica), tabular, icónico y sinóptico (mapas conceptuales).

De esta forma, y dada la complejidad que tiene el planteamiento de una sola tarea que satisfaga o evalúe ambos criterios al mismo tiempo, las tareas se seleccionaron de manera tal que, a lo largo del instrumento CNM-Antiderivada, se complementan para evaluar dichos aspectos. Otro factor relevante para la construcción de los ítems es la reconstrucción del significado holístico de la antiderivada (Gordillo y Pino-Fan, 2016). De acuer- 
do con D'Ambrosio (2013), la comprensión de los objetos matemáticos depende de la comprensión de cómo se originan estos y de sus motivaciones para el desarrollo.

\section{Herramientas metodológicas para el análisis de contenido de las tareas}

En este trabajo hemos adoptado los posicionamientos pragmatistas que nos brinda el marco teórico conocido como Enfoque Onto-Semiótico (eOS) del conocimiento y la instrucción matemática (Godino y Batanero, 1994; Godino, Batanero y Font, 2007). En el EOS se ha introducido una tipología de objetos matemáticos primarios: situaciones-problemas, lenguajes, definiciones, proposiciones, procedimientos y argumentos. Estos objetos matemáticos primarios están relacionados entre sí y forman redes de objetos intervinientes y emergentes de los sistemas de prácticas, lo que en el EOS se conoce con el nombre de configuraciones. Estas configuraciones pueden ser de tipo epistémicas (redes de objetos institucionales) o cognitivas (redes de objetos personales).

Así, para la realización de una práctica matemática y para interpretar que sus resultados son satisfactorios, se necesita poner en funcionamiento determinados conocimientos. Si consideramos, por ejemplo, los componentes del conocimiento para la realización y evaluación de la práctica que permite resolver una situación-problema (plantear y resolver un sistema de dos ecuaciones con dos incógnitas, por ejemplo), vemos el uso de lenguajes, verbales y simbólicos. Estos lenguajes son la parte ostensiva de una serie de conceptos, proposiciones y procedimientos, que intervienen en la elaboración de argumentos para decidir si las acciones simples que componen la práctica, y ella en cuanto que acción compuesta, son satisfactorias. En consecuencia, cuando un agente realiza y evalúa una práctica matemática, activa un conglomerado formado por situaciones-problemas, lenguajes, conceptos, proposiciones, procedimientos y argumentos, articulados en la configuración de la figura 1 (Font y Godino, 2006, p. 69).

La definición del objeto como emergente de los sistemas de prácticas y la tipología de objetos primarios responden a la necesidad de poder describir los sistemas de prácticas, con el fin de compararlos entre sí y tomar decisiones en el diseño, desarrollo y evaluación de procesos de enseñanza y aprendizaje de las matemáticas.

\section{Figura 1.}

Configuración de objetos matemáticos primarios.

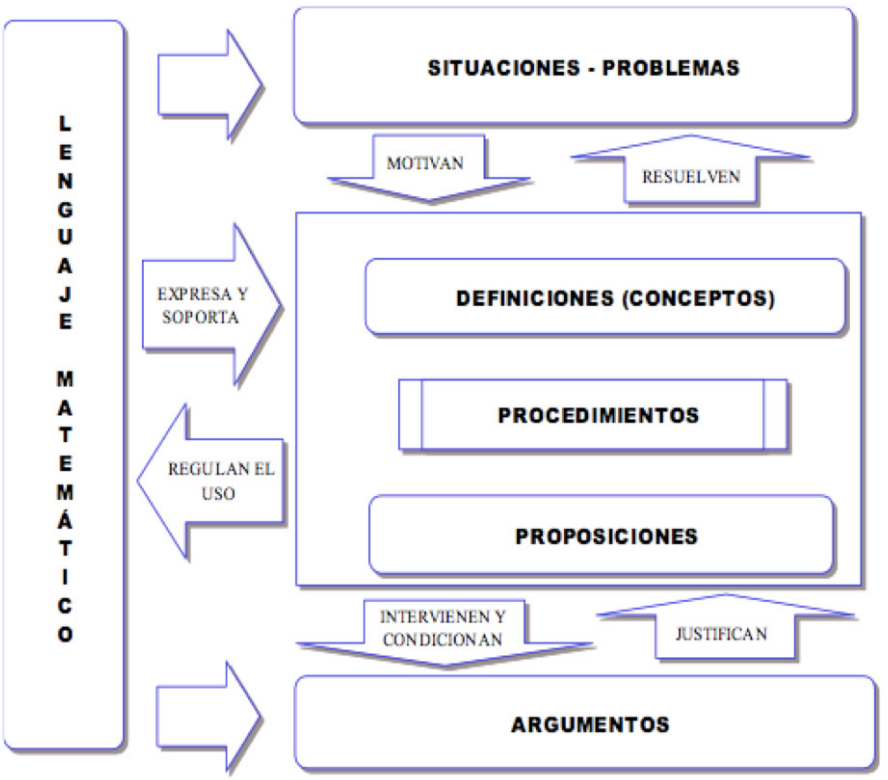

Fuente: elaboración propia. 
Estos objetos matemáticos primarios, que conforman la configuración, se manifiestan de diversas maneras durante la actividad matemática: el lenguaje con el cual nos referimos a ellos, a su vez, evoca conceptos o definiciones, que se vuelven operativos mediante procedimientos y propiedades asociadas que, a su vez, se manifiestan durante la solución de las tareas matemáticas. Además, cada uno de los objetos matemáticos primarios puede ser considerado desde distintas facetas o dimensiones duales (Godino, 2002): personal-institucional; ostensivo-no ostensivo; unitario-sistémico; expresión-contenido; extensivo-intensivo. Godino, Font, Wilhelmi y Lurduy (2011) señalan que tanto estas dualidades como los objetos matemáticos primarios pueden ser analizados desde una perspectiva proceso-producto, lo que lleva a considerar los siguientes procesos:

- Institucionalización-personalización.

- Generalización-particularización.

- Descomposición y análisis-composición y reificación.

- Materialización-idealización.

- Representación-significación.

La emergencia de los objetos matemáticos primarios, considerados en el modelo (ver figura 1), lleva asociada, respectivamente, los procesos de problematización, comunicación, definición, algoritmización, enunciación y argumentación. La figura 2 muestra el desglose, y las interacciones, de los objetos matemáticos primarios, las facetas duales desde las que estos pueden ser vistos y los procesos que llevan asociados.

\section{Figura 2.}

Configuración de objetos y procesos matemáticos.

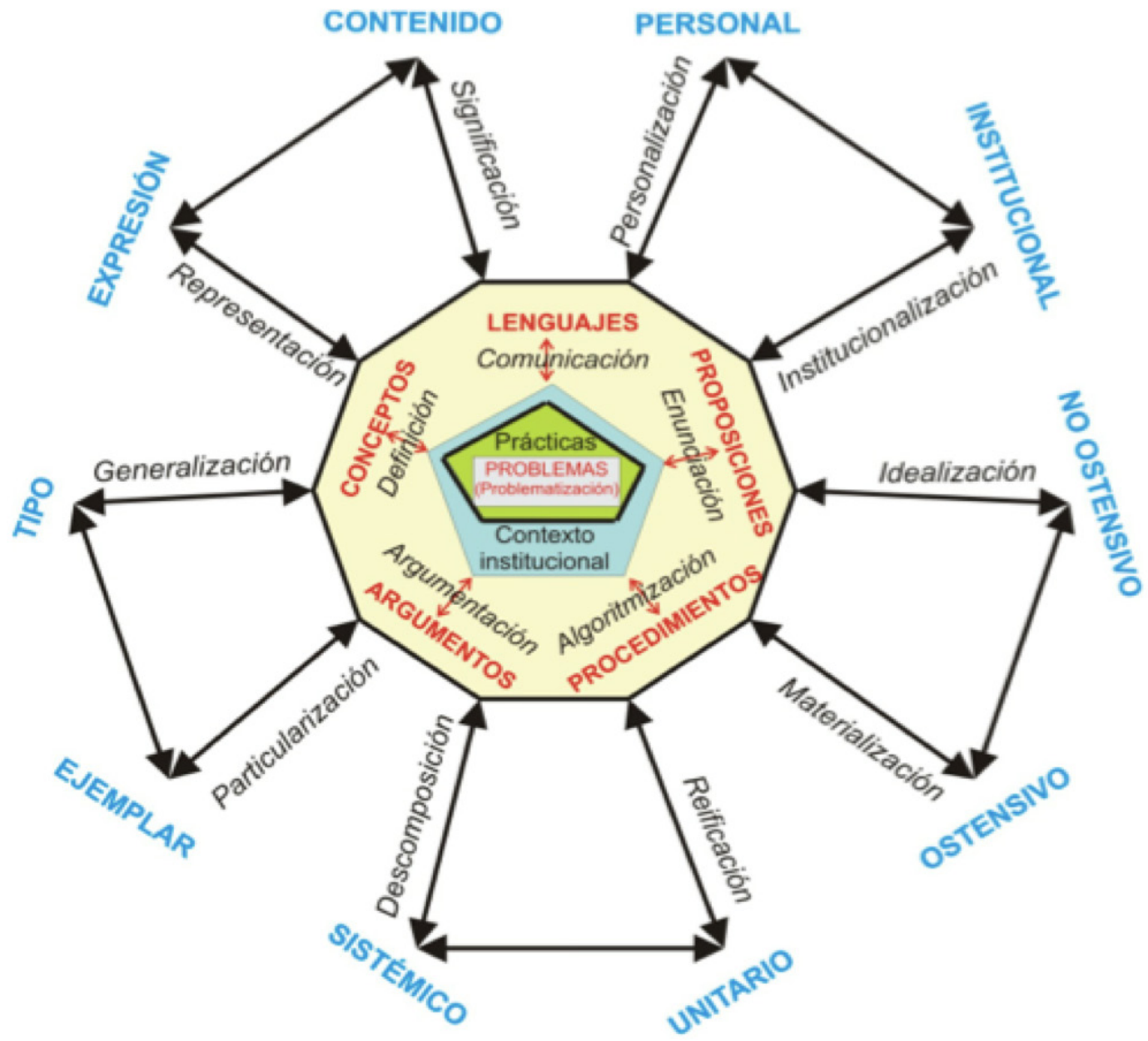

Fuente: elaboración propia. 
En esta figura se puede observar el papel central que tienen, en el EOS, las situaciones-problemas y las prácticas realizadas para resolverlas, así como su dependencia de los contextos institucionales en que estas tienen lugar.

Las redes de objetos y procesos que hemos descrito suelen recibir el nombre de configuración ontosemiótica (Pino-Fan, Godino y Font, 2015), y pueden ser de carácter epistémico o cognitivo, según se refiera a objetos y procesos matemáticos institucionales o personales, respectivamente. En este documento utilizaremos la herramienta configuración ontosemiótica epistémica para analizar detalladamente los contenidos que se movilizan en las prácticas necesarias para resolver las distintas tareas.

\section{Análisis del contenido que evalúan las tareas}

A continuación, se muestra el desglose del análisis de contenido de cada una de las tareas: tarea, solución plausible, análisis ontosemiótico, contenido curricular que evalúa la tarea y principales dificultades en la resolución.

\section{Tarea 1: significados verbales de la anti- derivada}

Esta tarea (ver figura 3) es una pregunta clásica que se ha hecho en diversas investigaciones (Badillo, 2003; Hähkiöniemi, 2006; Habre y Abboud, 2006; Bingolbali y Monaghan, 2008; Badillo, Azcárate y Font, 2011; Pino-Fan, Godino y Font, 2013) para explorar los significados que tienen los estudiantes de una noción matemática específica.

Figura 3.

Tarea 1: significados verbales de la antiderivada.

Tarea 1. ¿Qué significado o significados tiene para ti la antiderivada?

Fuente: elaboración propia.

\section{Solución plausible}

Al tratarse de una pregunta de carácter global, se espera que los estudiantes proporcionen alguno de los elementos de la lista de los posibles significados de la antiderivada, tales como:

- Es un procedimiento para obtener una familia de funciones, a partir de una función que ha sido derivada.

- Es la primitiva de la función $\mathrm{f}(\mathrm{x})$.

- Es la integral indefinida de una función $f(x)$.

\section{Análisis ontosemiótico}

Debido a la generalidad de la tarea (tanto la cuestión como el tipo de solución esperada), no se hará para esta el desglose operativo de las configuraciones de objetos y procesos del análisis epistémico. Basta con señalar que los elementos lingüísticos, conceptos, proposiciones y argumentos subyacentes a las posibles soluciones de la tarea son de carácter verbal (Pino-Fan, Godino y Font, 2013). Las descripciones verbales de los estudiantes no requieren conexiones entre los distintos significados de la antiderivada, pues les basta con recordar los usos y significados que han dado a dicho objeto a lo largo de su formación académica, para proporcionar su respuesta.

\section{Contenido curricular que se evalúa}

La tarea 1 evalúa las diversas acepciones de la antiderivada, personales e institucionales.

\section{Principales dificultades para su resolu- ción}

Es posible que la mayoría de los estudiantes respondan de forma errada: "proceso inverso de la derivada", sin recordar (o tener conocimiento) que la finalidad del proceso lleva a encontrar una familia de funciones a partir de una función que se ha derivado. 
Tarea 2: modelo sinóptico estructurado

Esta tarea (ver figura 4) se diseñó con el fin de determinar si los estudiantes organizan redes alrededor del objeto bajo estudio, en nuestro caso: la antiderivada. Esta organización jerárquica de redes de conceptos o mapas conceptuales es un instrumento de orden y de representación de los conocimientos.
Los mapas conceptuales pueden ser interpretados como modelos sinópticos de descripción estructurada de un sistema (Bencomo, Godino y Wilhelmi, 2004), y suelen ser representados mediante grafos, en cuyos vértices se colocan objetos. De esta manera, para la búsqueda de una respuesta a una cuestión, se organiza y representa el conocimiento mediante redes de objetos.

\section{Figura 4.}

Tarea 2: modelo sinóptico estructurado.

Tarea 2. Piensa en las siguientes expresiones: integral indefinida, $\frac{d y}{d x}$, velocidad, derivada, integral, área entre dos curvas, $f^{\prime}(x)$, antiderivada, $\int_{a}^{b} f(x) d x$, integral definida, teorema fundamental del cálculo. Elabora un mapa conceptual utilizando las expresiones anteriores y otras que tengas en la mente, y explica posibles relaciones entre ellas.

Fuente: elaboración propia.

\section{Solución plausible}

La figura 5 corresponde a una de muchas organizaciones de algunos términos del cálculo infinitesimal que pueden dar los estudiantes.

\section{Análisis ontosemiótico}

Debido a la diversidad de organizaciones que se pueden presentar, no se realiza para esta el desglose operativo de las configuraciones

Figura 5.

Un mapa conceptual de términos del cálculo infinitesimal.

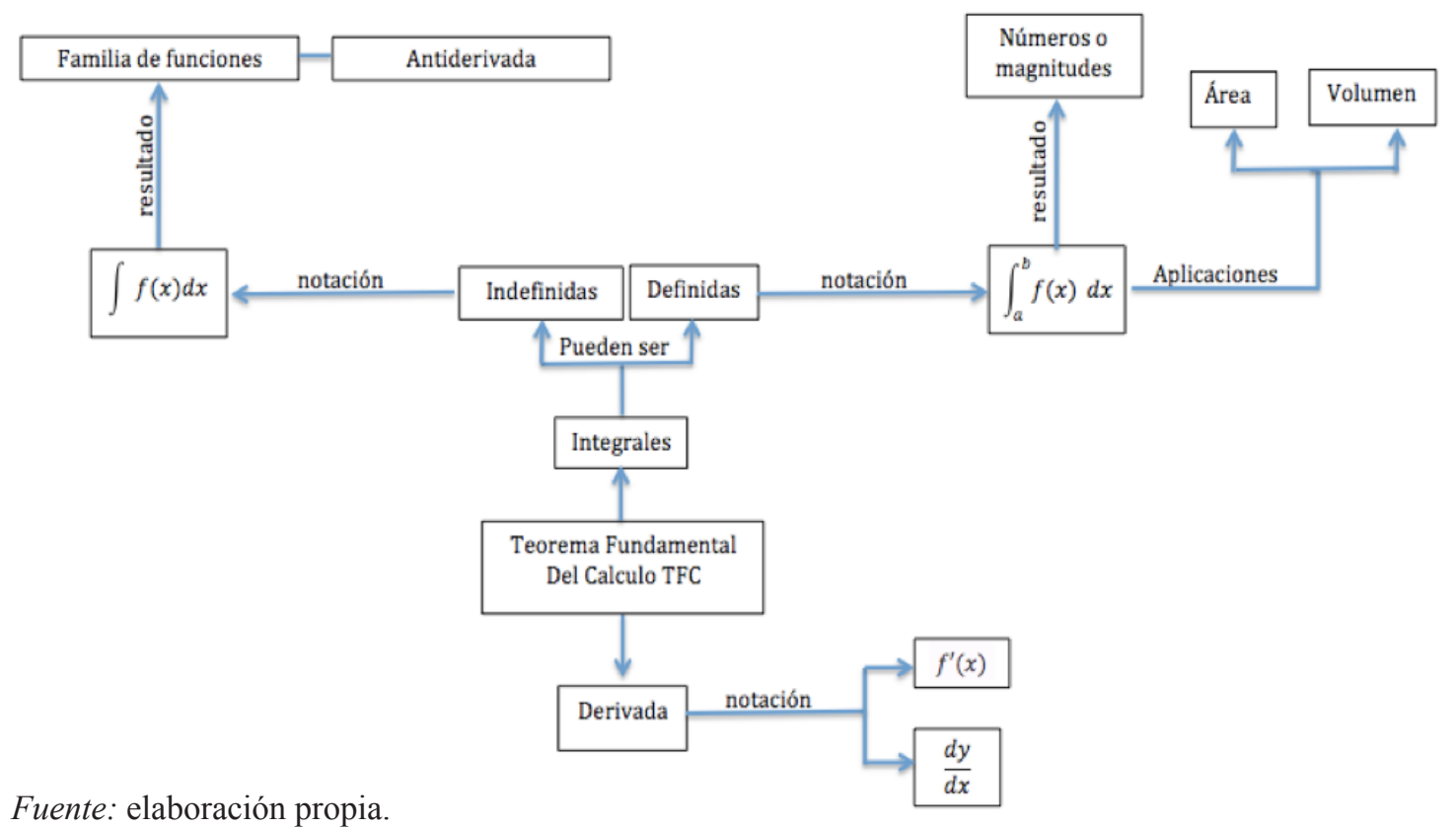


de objetos y procesos del análisis epistémico. Sin embargo, la construcción que presenten los estudiantes permitirá identificar conflictos epistémicos esenciales, tales como el aceptar o no la correspondencia entre las redes propuestas. Además, el análisis de los mapas conceptuales, desde el punto de vista del EOS, permite que se identifiquen las facetas duales de los elementos primarios que se ponen de manifiesto en este tipo de tareas, por ejemplo:

- La dualidad contenido-expresión puede identificarse en el uso de los conceptos y/o proposiciones en los diferentes registros.

- La dualidad personal-institucional, en el proceso intencional de hacer evolucionar el significado personal hacia el institucional pretendido.

- La dualidad ejemplar-tipo, en los argumentos de los estudiantes mediante ejemplos, al describirlos como representantes de una clase de objetos más general (formal).

- La dualidad no ostensivo-ostensivo, determinada por la necesidad del registro escrito para facilitar el contraste de las diversas producciones de los estudiantes.

- La dualidad sistémico-elemental, en el papel articulador que cumple la noción de antiderivada en todo el discurso, resaltando las conexiones matemáticas entre las entidades primarias involucradas.

\section{Contenido curricular que se evalúa}

La tarea 2 evalúa la organización de conceptos del cálculo infinitesimal y la determinación de relaciones entre las nociones dadas y otras nociones propuestas por el estudiante; en particular evalúa el reconocimiento del objeto de estudio como parte de un elemento de cálculo infinitesimal.

\section{Principales dificultades para su resolución}

Es posible que algunos estudiantes no agreguen nuevos términos a los dados en la tarea o que no se expliquen las conexiones entre los términos.

\section{Tarea 3: diferencia integral-antiderivada}

Esta tarea (ver figura 6) se diseñó de acuerdo con lo encontrado en el estudio histórico-epistemológico, realizado por Gordillo y Pino-Fan (2016). En él se encuentran diferencias conceptuales entre los objetos matemáticos integral y antiderivada. De esta forma, la tarea propuesta ayuda a explorar en los estudiantes la diferencia conceptual de estas dos nociones.

\section{Figura 6.}

Tarea 3: diferencia integral-antiderivada.

Tarea 3. ¿Existe alguna diferencia entre las nociones de integral y antiderivada? Justifica tu respuesta.

Fuente: elaboración propia.

\section{Solución plausible}

Una respuesta a la pregunta de la tarea propuesta es: sí, existe, pues la noción de integral es generalización de una suma de infinitos sumandos infinitamente pequeños en un intervalo. Generalmente, si la integral es definida, está asociada a los conceptos de área o volúmenes de sólidos, cuyos resultados particulares son números o cantidades de magnitud, mientras que la antiderivada es un procedimiento para obtener una familia de funciones a partir de una función que ha sido derivada, y en la que sus miembros difieren por una constante.

\section{Análisis ontosemiótico}

A continuación, se presenta un análisis de los elementos lingüísticos, conceptos y definiciones, propiedades y proposiciones, argumentos y procedimientos, y sus significados, que se espera sean activados en la solución de la tarea.

\section{Elementos lingüísticos}

La sentencia de la pregunta "existe diferencia..." implica un análisis que conlleve la determinación de "idea básica", en este 
caso, de dos objetos matemáticos del cálculo infinitesimal, integral y antiderivada, objetos matemáticos que comparten la misma simbología.

\section{Conceptos y definiciones}

- Integral: suma de sumandos infinitamente pequeños en un intervalo cerrado.

- Integral definida: dar límites al proceso de sumación en una función definida.

- Antiderivada: procedimiento para obtener una familia de funciones, a partir de una función que ha sido derivada.

\section{Propiedades y proposiciones}

- Para comprobar que la antiderivada de una función es correcta, basta con derivar.

- Al asignar un valor específico a la constante de "integración", se obtiene un miembro de una familia de funciones.

- Al obtener la familia de funciones de una función que ha sido derivada y seleccionar el miembro, cuya constante se hace cero (primitiva), se puede utilizar dicha primitiva para aplicar el Teorema Fundamental del Cálculo.

\section{Procedimientos}

Reflexivo-verbal: en este se describen relaciones o diferencias que se piensan sobre la integral y la antiderivada.

\section{Argumentos}

Verbal-deductivo: basado en los conceptos y definiciones sobre las dos nociones.

\section{Contenido curricular que se evalúa}

El contenido curricular que se evalúa con la tarea es la diferencia conceptual del objeto matemático integral y el objeto matemático antiderivada.

\section{Principales dificultades para su resolución}

En esta tarea se prevé que el estudiante no encuentre las diferencias entre estos dos ob- jetos y responda que no existen diferencias, que estas son lo mismo y los términos son sinónimos.

\section{Tarea 4: funciones elementales}

Esta tarea (ver figura 7) se diseñó de acuerdo con lo encontrado en el estudio histórico-epistemológico sobre la antiderivada (Gordillo y Pino-Fan, 2016). El recorrido histórico evidenció un significado parcial para la antiderivada, dado por Euler, al determinar que solo las sumas infinitas expresadas como funciones elementales poseen primitiva. En términos contemporáneos, lo anterior puede ser expresado como "dado el integrando de una función, si éste se puede expresar como función elemental, entonces tiene antiderivada". De no ser así, se debe acudir a métodos numéricos para calcular la integral en límites establecidos.

\section{Figura 7.}

Tarea 4: funciones elementales.

Tarea 4. ¿Es posible tener una función en $\mathbb{R}$, que se pueda integrar pero no tenga antiderivada? Justifica tu respuesta.

Fuente: elaboración propia.

\section{Solución plausible}

Una respuesta a la pregunta de la tarea propuesta es: sí, es posible. Una función que tiene antiderivada se puede expresar como función elemental, es decir, puede ser expresada como suma, resta, multiplicación, división o composición de otras funciones, usando un número finito de operaciones algebraicas. Un ejemplo es la función $\mathrm{f}(\mathrm{x})=\mathrm{x} /$ $(\mathrm{x}+1)$ : esta puede ser expresada como producto de otras dos, $\mathrm{h}(\mathrm{x})=\mathrm{x}$ y $\mathrm{g}(\mathrm{x})=1 /(\mathrm{x}+1)$ Hay funciones que no pueden ser expresadas como funciones elementales: por ejemplo, la función $f(x)=e^{x^{2}}$. Por lo tanto, con la expresión $\int e^{x^{2}} d x$ no es posible encontrar antiderivada. No obstante, sí es posible calcular la integral de la función con lími- 
tes definidos: por ejemplo, $\int_{1}^{5} e^{x^{2}} d x$, que se puede calcular por medio de integración numérica.

\section{Análisis ontosemiótico}

A continuación, se presenta un análisis de los elementos lingüísticos, conceptos y definiciones, propiedades y proposiciones, argumentos y procedimientos, y sus significados, que se espera sean activados en la solución de la tarea.

\section{Elementos lingüísticos}

- La expresión "función en $\mathbb{R}$ " equivale a un elemento transformador, en el que interviene un dominio y un recorrido que conlleva un conjunto numérico.

- La expresión "integrar" se refiere a un procedimiento matemático.

- La expresión "antiderivada" se refiere a un concepto matemático.

\section{Conceptos y definiciones}

- Funciones en $\mathbb{R}$ : estas se particularizan en aquellas que cumplan con la condición exigida en la tarea.

- Integral: dada como proceso es la suma de infinitos sumandos infinitamente pequeños.

- Funciones elementales: estas son entendidas como funciones; pueden ser expresada como suma, resta, multiplicación, división o composición de otras funciones, usando un número finito de operaciones algebraicas. Al ejemplificar una función, una de ellas puede ser la función: .

- Funciones transcendentes: estas son entendidas como funciones que transcienden el álgebra y no pueden ser expresadas como una función elemental. Al ejemplificar una función, una de ellas es la función: $f(x)=e^{x^{2}}$.

\section{Propiedades y proposiciones}

- Algunas funciones que tienen antiderivada se pueden expresar como función elemental.

- Hay funciones que no pueden ser expresadas como funciones elementales.

\section{Procedimientos}

- Reflexivo-verbal: en este se describen las diferencias entre relaciones o diferencias que se piensan sobre la integral y la antiderivada.

- Ejemplificación con casos particulares: a partir de ejemplos de funciones elementales y de funciones transcendentes, se puede dar respuesta a la tarea planteada.

\section{Argumentos}

- Empíricos: en estos se tratan de encontrar ejemplos para confirmar o refutar cierta hipótesis.

- Basado en los ejemplos de funciones elementales y transcendentes: son propuestos en los procedimientos y con los conceptos y definiciones dados anteriormente.

\section{Contenido curricular que se evalúa}

La tarea 6 evalúa los siguientes contenidos:

- Funciones elementales: concepto matemático asociado a un tipo de funciones que cumplen las condiciones de ser expresadas como suma, resta, multiplicación, división o composiciones de funciones.

- Integración numérica: en el sentido dado por un procedimiento para el cálculo de integrales definidas.

\section{Principales dificultades para su resolución:}

En esta tarea se prevé que el estudiante no responderá correctamente si no tiene conocimiento matemático sobre las funciones elementales y las funciones trascendentes, tal como se propone en la solución plausible. 
Tarea 5: aplicación de la antiderivada a la economía

La tarea 8 (ver figura 8) puede verse como una pregunta clásica de aplicación que se presenta en algunos libros de texto universitarios, con orientaciones hacia las ciencias económicas (Haeussler, Paul y Wood, 2008;
Arya y Lardner, 2004). Fue incluida para explorar la capacidad de relacionar la noción de antiderivada con otros objetos matemáticos en otros contextos. En este sentido, esta tarea es evaluadora de conocimiento y comprensión parcial de la noción matemática en contextos diferentes al matemático.

\section{Figura 8.}

Tarea 5: aplicación de la antiderivada en economía.

Tarea 5. En economía, la razón de cambio del costo total $\left(C_{t}\right)$ con respecto a la cantidad $q$ de unidades se llama Costo Marginal $\left(C_{M}\right)$, así $C_{M}=\frac{C_{t}}{d q}$; suponga que la función de costo marginal de un producto esta dada por $\frac{d c_{t}}{d q}=5 q^{2}-q$ ¿Determine la función de costo total?

Fuente: elaboración propia.

\section{Solución plausible}

Para determinar la función de costo total, dada una función de costo marginal, se deben separar las variables $C_{T}$ у $q$; así, $d C_{T}=\left(5 q^{2}-q\right) d q$. Verificamos que las funciones dadas sean elementales y procedemos a encontrar la antiderivada para cada uno de los elementos en la igualdad $\int C_{T} d q=\int\left(5 q^{2}-q\right) d q$, esto es, $C_{T}=\frac{5}{3} q^{3}-\frac{1}{2} q^{2}+C$. Esta ecuación proporciona el costo total $C_{T}$ de producir un producto con $q$ unidades; $C \in \mathbb{R}$ y $C$ representan el costo fijo de producir de la cantidad $q$ unidades.

\section{Análisis ontosemiótico}

A continuación, se presenta un análisis de los elementos lingüísticos, conceptos y definiciones, propiedades y proposiciones, argumentos y procedimientos, y sus significados, que se espera sean activados en la solución de la tarea.

\section{Elementos lingüísticos}

- La expresión "Determine la función...": sentencia que alude a un proceso de al- goritmización del cual emergerá un procedimiento para encontrar la función costo total.

- $\frac{\mathrm{dC}_{\mathrm{T}}}{\mathrm{dq}}=5 \mathrm{q}^{2}-\mathrm{q}$ : expresión simbólica que se refiere a la función de costo marginal.

- "q": letra que se refiere a una variable que representa la cantidad de unidades de lo que se produce.

- La letra $C_{T}$ : se refiere a la función de costo total.

\section{Conceptos y definiciones}

- Función de costo marginal: función que describe el incremento que sufre el costo cuando se incrementa la producción en una unidad, es decir, el incremento del costo total que supone la producción adicional de una unidad de un determinado bien.

- Función de costo total: función que da el costo que se tiene al producir una cierta cantidad de unidades de un producto.

- Antiderivada: procedimiento que permite obtener la función de costo total a partir de una función de costo marginal. 


\section{Propiedades y proposiciones}

- La derivada de la función de costo total es una función de costo marginal.

- La antiderivada de la función de costo marginal nos permite obtener la función de costo total.

\section{Procedimientos}

Método de variables separables para solución de ecuaciones diferenciales ordinarias de primer orden y primer grado. Dada una función de costo marginal, se deben separar las variables $C_{T}$ y $q$; así, $\mathrm{dC}_{\mathrm{T}}=\left(5 \mathrm{q}^{2}-\mathrm{q}\right) \mathrm{dq}$. Verificando que la función dada es elemental, procedemos a encontrar la antiderivada para cada uno de los elementos de la función.

\section{Argumentos}

A partir de la proposición, la antiderivada de la función de costo marginal permite obtener la función de costo total. Así, el costo total es el significado (institucional) que, en las ciencias económicas, se le confiere a la antiderivada de la función de costo marginal.

\section{Contenido curricular que se evalúa}

La tarea 8 evalúa los siguientes contenidos:

- Funciones y su aplicación en temas de la economía.

- Uso de las "reglas básicas de integración", entendidas como una técnica para encontrar la familia de funciones a partir de una función que ha sido derivada inicialmente.

- Aplicación de la antiderivada y sus propiedades en temas de la economía.

\section{Principales dificultades para su resolución}

Se prevé que el estudiante no identifique los símbolos y la notación, propios de la economía.

\section{Fiabilidad y validez de contenido de las} tareas

Con la finalidad de afianzar la fiabilidad y la validez del contenido de cada una de las tareas - validez entendida como la coherencia e integridad en el argumento e interpretación, como lo describe Kane (2013) _, estas se sometieron a revisión, mediante el juicio de expertos. Para el estudio se contactaron seis expertos ${ }^{1}$ del área de la educación matemática con especialidad en temas de cálculo, pertenecientes a las siguientes universidades: Universidad de Antioquia, Colombia (E1); Pontificia Universidad Católica de Valparaíso, Chile (E2); Universidad Autónoma de Querétaro, México (E3); Universidad de Sevilla, España (E4); Universidad de Barcelona, España (E5), y Universidad Autónoma de Barcelona, España (E6).

Para facilitar la colaboración de los participantes de este estudio, a cada uno de los expertos se le envío un formulario en el que podía opinar libremente, para cada una de las tareas, sobre el grado de relevancia de los siguientes aspectos:

- Distintos significados del objeto antiderivada.

- Representaciones activadas tanto en los enunciados como en las soluciones plausibles.

- Contenido matemático de las tareas en relación con el objeto antiderivada, es decir, el vínculo de la antiderivada con otras nociones matemáticas relevantes para su comprensión.

- Ausencia de algún contenido relevante.

- Redacción y comprensión de los enunciados.

Para cada uno de los puntos anteriores, los expertos podrían elegir una puntuación entre 1 y 5 , siendo 1 nada relevante y 5 totalmente relevante. Adicionalmente, se les proporcionó espacios en los que podían plasmar sus

${ }^{1}$ Para este estudio estamos utilizando la palabra "experto" en el sentido de Profesor Investigador asociado a una universidad, reconocido por la comunidad académica por sus publicaciones en revistas de alto impacto, registradas en bases de datos de tipo ISI/Web of Knowledge, SciElo, Scopus, ERIH, ZDM o Latindex, y con experiencia de más de 10 años en didáctica de cálculo. 
opiniones por tarea, y de todas las tareas en general. Las respuestas de los expertos contribuyeron a la mejora de las características y a la adecuación del nivel de dificultad de las tareas.

En general, las tareas fueron aprobadas y avaladas por parte de los expertos, tanto los enunciados como las soluciones plausibles de aquellas. La tabla 1 muestra la puntuación media otorgada por cada uno de los expertos.

Tabla 1.

Puntuación media de las tareas dada por los expertos

\begin{tabular}{|c|c|}
\hline Experto & Puntuación media \\
\hline E1 & 5,0 \\
\hline E2 & 4,8 \\
\hline E3 & 4,4 \\
\hline E4 & 4,2 \\
\hline E5 & 5,0 \\
\hline E6 & 5,0 \\
\hline Puntuación en escala (1 a 5); Media $=4,722$ \\
\hline
\end{tabular}

Fuente: elaboración propia.

Para determinar la calificación de cada experto, se promedió la puntuación asignada a cada uno de los criterios evaluados para cada una de las tareas. Como se puede observar, la media total obtenida fue de 4,72 puntos. En la versión definitiva de las tareas se incluyeron las sugerencias y recomendaciones de cada uno de los expertos; estas recomendaciones estaban orientadas hacia la mejora de la redacción de algunos enunciados y hacia la consideración de algunos elementos adicionales en las soluciones de las tareas. Los elementos de recomendación corresponden a la dificultad que tiene un estudiante para resolver la tarea propuesta, y fueron anexados en el análisis ontosemiótico.

Para que la fiabilidad y validez de contenido de cada una de las tareas fueran lo más altas posibles, además de las sugerencias de ex- pertos, se realizaron, por tarea, los análisis de contenido detallados, que se presentaron en la sección 3. Dichos análisis, que denominamos análisis ontosemióticos, junto con las herramientas teórico-metodológicas contempladas para llevar a cabo este tipo de estudio, han sido sugeridos y utilizados con el mismo fin en diversos estudios (Pino-Fan y Font, 2015; Pino-Fan, Godino y Font, 2013), y se prevé su potencialidad a la hora de caracterizar y tener un buen grado de validez de contenido en el diseño de tareas como el que aquí presentamos.

\section{Reflexiones finales}

En esta investigación, presentamos el diseño de algunas tareas que nos permiten evaluar y caracterizar el conocimiento y las prácticas matemáticas sobre la antiderivada, en estudiantes de los primeros cursos universitarios. La noción de conocimiento (conocimiento matemático), desde un punto de vista pragmatista como el adoptado por el EOs, incluye y vincula las actividades de comprensión, competencia y disposición, que intervienen en las prácticas matemáticas desarrolladas con la finalidad de resolver un problema. Esta forma pragmática de entender el conocimiento ha sido considerada en el diseño de cada una de las tareas, toda vez que estas requieren, para su resolución, de la movilización congruente, tanto de los diversos registros de representación para la antiderivada (Duval, 1995; 2006a) como de la diversidad de significados parciales de dicha noción matemática (Gordillo y Pino-Fan, 2016).

Las posibles dificultades en la resolución de las tareas y el análisis ontosemiótico (contenido y curricular) realizado para cada una ellas es anterior a la aplicación de estas. Además, permite observar, describir y predecir la actividad como un complejo conjunto de prácticas matemáticas, realizada por estudiantes universitarios al resolver las tareas propuestas, alrededor del objeto matemático. En dichas prácticas se pueden identificar la configuración de objetos y procesos matemáticos primarios, propuestos por el marco 
teórico del Eos, que se ha denominado análisis ontosemiótico.

Este análisis ontosemiótico, utilizado en cada una de las tareas, se prevé como una herramienta potente para poder identificar, analizar y tener un buen grado de validez de contenido. Herramienta teórico metodológicas del EOS, que es validada y utilizadas en otros diseños de tareas (Pino-Fan y Font, 2015; Pino-Fan, Godino y Font, 2015).

Por otro lado, añadido a lo anterior, el estudio mediante juicio de expertos ha dado evidencia de que el diseño de cada una de las tareas, en efecto, evalúa lo que se previó en los análisis de contenido, y que se realiza para cada una de ellas. Queda así sustentado el hecho de que el instrumento diseñado y descrito a lo largo de este artículo sí evalúa la articulación de los significados institucionales y personales, respecto a la antiderivada, lo cual proporciona, entonces, argumentos válidos para determinar que cada una de las tareas es evaluadora de conocimiento y comprensión parcial, y en su globalidad: evaluadora de comprensión, competencia y disposición de la noción antiderivada.

\section{Reconocimientos}

Este artículo se ha desarrollado en el marco del Proyecto de Investigación FONDECYT, de iniciación No 11150014, financiado por la Comisión Nacional de Investigación Científica y Tecnológica (CONICYT) de Chile.

\section{Referencias}

Artigue, M. (1995). Ingeniería didáctica. En Gómez, P. (Ed.) Ingeniería didáctica en educación matemática. 97-140. México: Grupo Editorial Iberoamérica.

Arya, J. C. y Lardner, R. W. (2004). Matemáticas aplicadas a la administración y a la economía. México: Pearson Educación.

Badillo, E. (2003). La derivada como objeto matemático y como objeto de enseñanza y aprendizaje en profesores de matemáticas de Colombia. (Tesis doctoral, Universitat Autònoma de Barcelona, Barcelona, España).
Badillo, E., Azcárate, C., \& Font, V. (2011). Análisis de los niveles de comprensión de los objetos f'(a) y f'(x) en profesores de matemáticas. Enseñanza de las Ciencias, 29(2), 191-206.

Bencomo, D., Godino, J. D., \& Wilhelmi, M. (2004). Elaboración de redes ontosemióticas de configuraciones didácticas con atlas/ ti. En Cañas, A. J, Novak, J. D. \& González, F. M. (Eds.). Concept Maps: Theory, Methodology, Technology. 2, 71-74. Pamplona: Universidad Pública de Navarra.

Bingolbali, E., \& Monaghan, J. (2008). Concept image revisited. Educational Studies in Mathematics, 68(1), 19-35.

Crisostomo, E. (2012). Idoneidad de Procesos de estudio del cálculo integral en la formación de profesores de matemáticas: Una aproximación desde la investigación en didactica del cálculo y el conocimiento profesional. (Tesis Doctoral, Universidad de Granada, España).

Czarnocha, B., Loch, S., Prabhu, V. \& Vidakovic, D. (2001). The concept of definite integral: coordination of two schemas. In M. van den Heuvel-Panhuizen (Ed.), Proceedings of the 25th conference of the International Group for the Psychology of Mathematics Education (vol. 2, pp. 297-304). Utrecht, The Netherlands: Freudenthal Institute.

D’Ambrosio, U. (2013). Priorizar história e filosofia da matemática. Cuadernos de Investigación y Formación en Educación Matemática, 8(11),175-186. Disponible en: $<$ http://revistas.ucr.ac.cr/index.php/cifem/ article/view/14724/13969>.

Depool, R. A. (2004). La enseñanza y aprendizaje del cálculo integral en un entorno computacional. Actitudes de los estudiantes hacia el uso de un programa de cálculo simbólico (pcs). (Tesis doctoral, Universidad de La Laguna, España).

Doorman, M., \& Maannen, J. V. (2008). A historical perspective on teaching and learning calculus. Australian Senior Mathematics Journal, 22(2), 4-14. 
Duval, R. (1995). Sémiosis et pensée: registres sémiotiques et apprentissages intellectuels. Berne, Switzerland: Peter Lang.

Duval, R. (2006a). A cognitive analysis of problems of comprehension in a learning of mathematics. Educational Studies in Mathematics, 61(1-2), 103-131.

Duval, R. (2006b). Un tema crucial en la educación matemática: la habilidad para cambiar el registro de representación. La Gaceta de la RSME, 9(1), 143-168.

Font, V. (1999). Procediments per obtenir expressions simbòliques a partir de gràfiques. Aplicacions a la derivada. (Tesis doctoral, Universitat de Barcelona, España).

Font, V. (2001). Processos mentals versus competència, Biaix, 19, 33-36.

Font, V., \& Godino, J. D. (2006). La noción de configuración epistémica como herramienta de análisis de textos matemáticos: su uso en la formación de profesores. Educação Matemática Pesquisa, 8(1), 67-98.

Godino, J. D. (2002). Un enfoque ontológico y semiótico de la cognición matemática. Recherches en Didactique des Mathématiques, 22(2/3), 237-284.

Godino, J. D., \& Batanero, C. (1994). Significado institucional y personal de los objetos matemáticos. Recherches en Didactique des Mathématiques, 14(3), 325-355.

Godino, J. D., Batanero, C., \& Font, V. (2007). The onto-semiotic approach to research in mathematics education. ZDM. The International Journal on Mathematics Education, 39 (1), 127-135.

Godino, J. D., Font, V., Wilhelmi, M., \& Lurduy, O. (2011). Why is the learning of elementary arithmetic concepts difficult? Semiotic tools for understanding the nature of mathematical objects. Educational Studies in Mathematics, 77(2), 247-265.

Gordillo, W., \& Pino-Fan, L. (2016). Una propuesta de reconstrucción del significado holístico de la antiderivada. Bolema, 30(55), 535-558. doi: 10.1590/1980-4415v30n55a12

Gray, E., \& Tall, D. (1994). Duality, ambiguity, and flexibility: a "proceptual" view of simple arithmetic. Journal for Research in Mathematics Education, 25(2), 116-140. doi: $10.2307 / 749505$

Habre, S., \& Abboud, M. (2006). Student's conceptual understanding of a function and its derivative in an experimental calculus course. Journal of Mathematical Behavior, 25(1), 52-72.

Haeussler, E. F., Paul, R. S. \& Wood, R. J. (2008). Matemáticas para administración y economía. México: Pearson Educación.

Hähkiöniemi, M. (2006). The role of representations in learning the derivative. (Tesis doctoral, University of Jyväskylä, Finland).

Kane, M. (2013). Validating the interpretations and uses of test scores. Journal of Educational Measurement, 50(1), 1-73. doi: 10.1111 / jedm. 12000

Kintsch, W. (1998). Comprehension: a paradigm for cognition. Cambridge: Cambridge University Press

Labraña, P. A. (2001). Avaliación das concepcións dos alumnos de COU e Bachalerato acerca do significado do Cálculo Integral definida. (Tesis doctoral, Universidad de Santiago de Compostela, España).

Metaxas, N. (2007). Difficulties on understanding the indefinite integral. En Woo, J. H., Lew, H. C., Park, K. S. \& Seo, D. Y. (Eds.), Proceedings of the 31st Conference of the International Group for the Psychology of Mathematics Education, (Vol. 3, pp. 265-272). Seoul, Korea: PME.

Pino-Fan, L. (2014). Evaluación de la faceta epistémica del conocimiento didáctico matemático de futuros profesores de bachillerato sobre la derivada. Granada: Universidad de Granada. 
Pino-Fan, L., \& Font, V. (2015). A methodologolgy for the desing of questionnaries to explore relevant aspects of didactic-mathematical knowledge of teachers. In Beswick, K., Muir, T., \& Wells, J. (Eds.), Proceedings of the 39th Conference of the International Group for the Psychology of Mathematics Education. (Vol. 4, pp. 25-32). Hobart, Australia: PME.

Pino-Fan, L., Godino, J. D., \& Font, V. (2010). Conocimiento didáctico-matemático sobre la enseñanza y aprendizaje de la derivada. Memorias XIII Escuela de Invierno en Matemática Educativa, Instituto Tecnológico y de Estudios Superiores de Monterrey, Monterrey, Nuevo León, México. 206-213.

Pino-Fan, L., Godino, J. D., \& Font, V. (2011). Faceta epistémica del conocimiento didáctico- matemático sobre la derivada. Educação Matemática Pesquisa, 13(1), 141178.

Pino-Fan, L., Godino, J. D. \& Font, V. (2013). Diseño y aplicación de un instrumento para explorar la faceta epistémica del conocimiento didáctico-matemático de futuros profesores sobre la derivada ( $1^{\mathrm{a}}$ Parte). REVEMAT, 8(2), p. 1-49.

Pino-Fan, L., Godino, J. D., \& Font, V. (2015). A methodology for the design of questionnaires to explore the mathematical dimension and the epistemic facet of didactic-mathematical knowledge of teachers. CERME 9, WTG 20: Mathematics teacher knowledge, beliefs and identity. Recuperado de http://www.cerme9.org/products/twg20/

Pirie, S. \& Kieren, T. (1994). Growth in mathematical understanding: How can we characterise it and how can we represent it? Educational Studies in Mathematics, 26(23)165-190. doi: 10.1007/BF01273662

Ponce-Campuzano, J. C., \& Rivera-Figueroa, A. (2011). Unexpected results using computer algebraic systems for computing antiderivates. Far East Journal of Mathematical Education, 7(1), 57-80.
Robles, M. G., Del Castillo, A. G., \& Font, V. (2012). Análisis y valoración de un proceso de instrucción sobre la derivada. Educación matemática, 24(1), 3571. Recuperado de http://www.scielo. org. $\mathrm{mx} / \mathrm{scielo}$.php? script=sci_arttext\&pi$\mathrm{d}=$ S1665-58262012000100003\&lng=es\&tl$\mathrm{ng}=\mathrm{es}$.

Sfard, A. (1991). On the dual nature of mathematical conceptions: reflections on processes and objects as different sides of the same coin. Educational Studies in Mathematics, 22, 1-36. doi: 10.1007/BF00302715

Tall, D. (1991). Advanced mathematical thinking. Dordrecht: Kluwer, A. C.

Tall, D. (2009). Dynamic mathematics and the blending of knowledge structures in the calculus. ZDM Mathematics Education, 41(1), 481-492. 